\title{
Development and psychometric evaluation of the Anticipated Food Scarcity Scale (AFSS)
}

Michał Folwarczny ${ }^{1}$, Norman P. Li ${ }^{2}$, Valdimar Sigurdsson ${ }^{1}$, Lynn K. L. Tan ${ }^{2}$, and Tobias Otterbring $^{3}$

\author{
${ }^{1}$ Department of Business Administration, Reykjavik University \\ ${ }^{2}$ School of Social Sciences, Singapore Management University \\ ${ }^{3}$ Department of Management, University of Agder
}

\begin{abstract}
Author Note
Michał Folwarczny (iD https://orcid.org/0000-0002-1686-4933
\end{abstract}

Correspondence concerning this article should be addressed to Michał Folwarczny, Department of Business Administration, Reykjavik University, Menntavegur 1, 101 Reykjavik, Iceland. E-mail: michalf@tuta.io

This project was funded by the Icelandic Research Fund (Doctoral Student Grant to Michal Folwarczny number 206880-051). 


\begin{abstract}
Mass media extensively inform societies about events threatening the global food supply (e.g., pandemics or Brexit). Consumers exposed to such communication may perceive food resources as becoming scarcer. In line with the evolutionary account, these perceptions can shift decision-making in domains such as food preferences or prosociality. However, the current literature has solely focused on actual and past food insecurity experiences threatening mostly low-income families, thus neglecting the future-oriented perceptions among the general population. This paper broadens the food insecurity research scope by developing a new construct — anticipated food scarcity (AFS) — which is defined as the perception that food resources are becoming less available (in the future). We have developed and psychometrically validated the 8-item Anticipated Food Scarcity Scale (AFSS) in eight studies $(N=1333)$. The 8-item AFSS is unidimensional and has good psychometric qualities. The scale is sensitive to food scarcity cues and, therefore, can be used in experimental research. Moreover, the scale's relatively narrow set of items makes it an exceptionally potent tool for use in online surveys, field settings, and lab studies. Taken together, the AFSS presents an alternative approach to food scarcity measurement in affluent societies and, consequently, can foster novel research on food waste, sustainability, prosocial behaviors, and other similar topic areas.

Keywords: food scarcity, food shortages, food insecurity, the insurance hypothesis, evolutionary mismatch
\end{abstract}




\section{Development and psychometric evaluation of the Anticipated Food Scarcity Scale (AFSS)}

\section{Introduction}

Amid the COVID-19 pandemic, people around the world have been panic-buying food (Benker, 2021). According to a BBC report (Lufkin, 2020), in Singapore, consumers grabbed up lots of rice and instant noodles, leading to shortages of these products in supermarkets. In Auckland, shoppers spent $40 \%$ more on groceries than they did a year ago. Similar behaviors have been observed in the past, such as during the 1962 Cuban missile crisis and in preparation for Y2K, when Americans stockpiled canned foods in their basements (Lufkin, 2020). Experts have warned that Brexit may trigger even more intense panic buying among consumers (Casalicchio, 2020). These shopping sprees often result in excessive food waste. Crucially, the United Nations Development Programme (UNDP, 2020) points at limiting unnecessary consumption as one of the foremost steps in achieving sustainable development.

Despite the pervasiveness of this phenomenon and the importance of its consequences, predictors of food hoarding behaviors amidst pandemics or political disputes are not fully understood. Although such events usually do not cause actual food shortages in developed countries, it is plausible that receiving information about such turmoil may nonetheless induce consumers to anticipate food scarcity. As a result, consumers may purchase, consume, or waste more food than usual due to unjustified food stockpiling (see Nettle et al., 2017, for an overview of the role that cues to food scarcity play in food decision-making) and consumption (Folwarczny et al., 2021). To investigate this possibility, a scale measuring anticipated food scarcity (AFS) is vital. Existing tools used in affluent countries are typically retrospective and focus on the actual insufficiency of food resources (e.g., Ashby et al., 2016; Nettle et al., 2019). For example, an established tool for measuring food insecurity-HFIAS - focuses on the frequency of experiencing food unavailability in the past (Coates et al., 2007). In other words, these tools focus on the 
actual experience of food shortages in specific populations. What is missing is a tool to measure expectations of impending food shortage. Accordingly, we developed the Anticipated Food Scarcity Scale (AFSS) to capture perceptions of food resources becoming less available in the future among the general population.

Our focal construct differs from the existing literature (cf. Ashby et al., 2016) in that it focuses on an increasing scarcity of food resources rather than on food insecurity, which tends to denote emotions such as fear and anxiety (Merriam-Webster, 2021). Indeed, the results presented in Table 3 suggest that AFS and affective states are only weakly related.

\section{Anticipated food scarcity (AFS)}

Major global concerns such as climate change, water scarcity, or resource depletion will have more severe consequences for food resources in the future than in the present (Hanjra \& Qureshi, 2010). Hence, our construct of interest is future-oriented. When defining the construct, we draw from work on past experiences of food insecurity by Coates et al. (2007). As such, in the remainder of this article, we define AFS as "the perception of future food resources becoming insufficient in terms of accessibility and availability (e.g., certain foods might disappear or become less affordable)." Availability refers to the physical existence of food resources (e.g., food production), whereas accessibility refers to individuals' ability to obtain food (e.g., having enough money for groceries). Webb et al. (2006) discuss how these dimensions are understood in the literature on food insecurity.

This current perception of future food scarcity relies on past experiences of food unavailability, beliefs concerning the influence of various worldly events and factors, and beliefs in wielding power over others. As AFS is a perception, its magnitude can vary over time. In sum, whereas AFS is informed by top-down processes such as present knowledge about future food production or beliefs that one can control what happens in politics, it is also informed by bottom-up processes such as exposure to cues related to food scarcity. Importantly, food scarcity cues may influence our immediate reactions regardless of whether they suggest food scarcity that is distant in time and space or more proximate 
(e.g., neighbors who have to rely on food stamps).

\section{Perceivable cues related to food scarcity}

To understand why this is so, we need to consider basic principles of evolutionary mismatch (Li et al., 2018; Tooby \& Cosmides, 1990). That is, the mechanisms that comprise human psychology evolved to cope primarily with immediate threats and opportunities (e.g., a lion approaching, a drought that leads to famine, an attractive person who appears available). Because our ancestors did not routinely face technology-induced processes such as global warming that have consequences many years later, our threat-handling mechanisms did not evolve to differentiate cues that indicate immediate dangers versus those that suggest much more distant dangers (Griskevicius et al., 2012; Ornstein \& Ehrlich, 2000). Hence, psychological mechanisms respond to most threat cues as if they were indicating an impending danger. Accordingly, cues related to evolutionarily novel threats that are distant in time (e.g., food scarcity occurring this year vs. food scarcity occurring in three decades) or space (e.g., one's local neighborhood vs. a country in another continent) may similarly activate mechanisms that produce immediate responses to mitigate those threats in the current environment (cf. Li et al., 2018).

In this regard, exposure to content describing food scarcity in the distant future may shift decision-making processes in humans in a manner similar to the experience of either impending or actual food unavailability. Crucially, whereas actual food unavailability always entails the physical experience of food insufficiency such as hunger, anticipated food scarcity induced by, for instance, reports about Brexit does not necessitate physical experiences. As such, AFS is distinct from food insecurity as it is defined in the current literature. According to the Food and Agriculture Organization of the United Nations (FAO), "Food security exists when all people, at all times, have physical and economic access to sufficient, safe and nutritious food to meet their dietary needs and food preferences for an active and healthy life" (FAO, 1996). This definition does not encompass any form of anticipation and perceptions about future food availability. Hence, any tool 
based upon this definition will inevitably capture a different construct than AFS and will not be predictive of responses to future food scarcity cues.

In line with this reasoning, a recent study has suggested that exposing participants to videos showing food scarcity induced by climate change occurring in distant parts of the world increases their current preferences toward energy-dense foods while decreasing their preferences toward low-calorie alternatives (Folwarczny et al., 2021). These results support the view that psychological mechanisms have not evolved to differentiate between current and future threats and imply that exposure to any cues related to food scarcity may shift food preferences toward energy-dense products. However, tools measuring related constructs, such as the United States Department of Agriculture's Food Security Survey Module (FSSM), are typically retrospective and primarily capture food insecurity defined as the physical unavailability of food resources (Ashby et al., 2016). Thus, these tools cannot predict a shift in food preferences resulting from exposure to food scarcity cues, especially if these cues are related to scarcity distant in time (i.e., happening in two decades) and space (i.e., happening on another continent).

\section{Media exposure and AFS}

Many events can serve as perceivable cues to upcoming food scarcity, especially when mass media vividly show them. People across the globe have been extensively informed about lockdowns imposed as a response measure to control the spread of the COVID-19 pandemic. These lockdowns have triggered labor deficiencies and thus insufficient food production (Galanakis, 2020). Climate change-another issue causing heated debates in media - threatens the global food supply chain, but its effects are much slower than the effects of pandemics (Wheeler \& Von Braun, 2013). Brexit is yet another prominent event that can make consumers perceive future food resources as scarcer, at least in the United Kingdom (Lang \& McKee, 2018). In short, numerous events pose a risk of upcoming food scarcity over time. Notably, people who are not at risk of direct exposure to such events may overestimate related threats (in particular, their immediacy) due to, for 
instance, media communication with photographs depicting large crowds or diminishing food supplies (e.g., Garfin et al., 2020). However, as described above, the current literature lacks a scale that can gauge the extent to which events such as trade wars affect the perceptions associated with future food scarcity.

\section{Research aims and overview of the studies}

Thus far, no scale has been developed to capture the perception that future food resources are becoming scarcer. We address this critical shortcoming by developing and psychometrically validating the 8-item AFSS.

Studies 1a-1c aimed to develop the preliminary items' pool for subsequent psychometric evaluation. Study 2 was conducted to shorten the scale to a desirable length and to assess its psychometric properties. Studies 3a and 3b confirmed the scale's unidimensionality and examined its convergent and discriminant validity to rule out the possibility that AFS is a ramification of general anxiety or other emotional states. In line with the evolutionary account, Study 3b revealed that AFS might be linked to prosociality. We conducted Study 4 to confirm that the specific, distant timeframes mentioned in the instructions do not affect the scale's psychometric properties or averaged scores. Although several items in the final AFSS (see Table 2) refer to predictability rather than mere scarcity of food resources, analyses reported in Studies 2-4 clearly show that the scale is unidimensional; hence these items capture the same construct. Finally, as the scale was developed primarily for experimental research, Study 5 showed that the AFSS is sensitive to experimentally manipulated food scarcity. Table 1 depicts an overview of the scale development process. 


\section{Method}

\section{Analytical approach}

We applied nonparametric item response theory to evaluate the AFSS psychometric properties. The advantage of nonparametric item response theory over parametric item response theory is that nonparametric item response theory makes fewer assumptions about the distribution of latent variables and requires smaller sample sizes (Sijtsma \& Molenaar, 2002). Parametric models assume that latent variables are continuous, and this may not always be the case in social sciences.

For nonparametric item response theory, we performed a Mokken scale analysis (MSA) (Van der Ark, 2007, 2012). A Mokken scale analysis investigates whether responses to scale items correspond to an increasing level of the latent variable of interest (Van Schuur, 2003). Specifically, we tested the scale unidimensionality, monotonicity, local independence, and invariant item ordering. If the first three criteria are met, then the tested set of items meets the requirements for a monotone homogeneity model and can be considered a scale, whereas meeting the invariant item ordering criterion (nonintersecting item response functions) is required for the double monotonicity model to hold (Mokken, 1971; Van der Ark, 2007, 2012). We investigated unidimensionality - which is the quality of a set of items that enables them to be located on a continuum of the hypothesized latent variable according to the probability of obtaining high total scores (Van Schuur, 2003) - by evaluating the coefficients of homogeneity $H$ that range from 0 to 1 . The former number suggests no association between the items, whereas the latter number suggests a perfect association between them (Van der Ark, 2007). We also applied the automated item selection procedure (AISP) to further explore scale unidimensionality. This algorithm positions items into scales at increasing homogeneity thresholds (Hemker et al., 1995). If several items start forming another scale at a particular homogeneity threshold, this suggests that a scale may have more than one dimension (Dima, 2018). Monotonicity, local independence, and invariant item ordering were scrutinized separately with tools provided 
in the "Mokken" package (Van der Ark, 2007). Table 1 illustrates the scale development process across all the studies.

We performed all the analyses in $\mathrm{R}$. Clean data sets, the data analysis code, the $\mathrm{R}$ markdown files, Supplementary Information, and additional analyses following the recently developed - and modified for this research - six-step psychometric protocol in R (Dima, 2018) have been made publicly available through the Open Science Framework (OSF; https://osf.io/kqf3g/). All the studies have been programmed in PsyToolkit (Stoet, 2010, 2017), and the source code has been made available therein.

We collected data in full compliance with the Declaration of Helsinki and the American Psychological Association's guidelines on research involving human subjects. The studies complied with the local regulations, and a formal Institutional Review Board's approval was neither needed nor sought. However, all participants approved an informed consent form before the studies. Data were collected between March 2020 and February 2021. Only participants whose Prolific submission acceptance rate was $99 \%$ and higher and who had not taken part in any of our earlier experiments were included to ensure high data quality. We have performed and reported several data exclusions in Studies 2 and 3a, but including all participants in datasets yielded similar results. 


\section{Table 1}

Overview of studies included in the AFSS development process

\begin{tabular}{|c|c|c|c|}
\hline Study & Aim & $N$ & Measures \\
\hline 1a & Develop the initial items pool & 10 & Proposed items \\
\hline $1 b$ & $\begin{array}{l}\text { Investigate the face validity of the } \\
\text { items and reduce their number }\end{array}$ & 26 & $\begin{array}{l}\text { Face validity, comprehension, and } \\
\text { grammatical correctness }\end{array}$ \\
\hline $1 \mathrm{c}$ & Pretest the first scale, reword items & 52 & AFSS \\
\hline 2 & $\begin{array}{l}\text { Shorten the scale, evaluate its' psy- } \\
\text { chometric properties }\end{array}$ & 303 & AFSS \\
\hline $3 a$ & $\begin{array}{l}\text { Refine the scale, test the convergent } \\
\text { and discriminant validity }\end{array}$ & 212 & $\begin{array}{l}\text { AFSS, FIES, Radimer/Cornell, } \\
\text { Mini-K, HEXACO- } 60 \text { (emotional- } \\
\text { ity), Generalized Sense of Power, } \\
\text { Locus of Control }\end{array}$ \\
\hline $3 b$ & $\begin{array}{l}\text { Further test the convergent and dis- } \\
\text { criminant validity }\end{array}$ & 140 & $\begin{array}{l}\text { AFSS, PANAS, STAI, support for } \\
\text { Universal Basic Income, attitude to- } \\
\text { ward welfare recipients }\end{array}$ \\
\hline 4 & $\begin{array}{l}\text { Investigate the scale's psychometric } \\
\text { properties under different instruc- } \\
\text { tions, test the criterion validity }\end{array}$ & 415 & $\begin{array}{l}\text { AFSS, calorie estimates, food prefer- } \\
\text { ences }\end{array}$ \\
\hline 5 & $\begin{array}{l}\text { Test whether the scale is sensitive to } \\
\text { food scarcity cues }\end{array}$ & 175 & AFSS, I-PANAS-SF \\
\hline
\end{tabular}

Note. Participants in all the studies were recruited through Prolific. They were native English speakers, mostly from the United States and the United Kingdom. Participants could not take part in more than one study. 


\section{Study 1a: Item development}

We aimed to develop at least three times more items than the desired number for the final scale (Boateng et al., 2018). First, we reviewed the academic literature and think tank reports on future food scarcity. Recent reports by the Food and Agriculture Organization of the United Nations (FAO, 2016), the Centers for Disease Control and Prevention (CDC, 2019), and the Center for Strategic and International Studies (Sova et al., 2019) served as a basis for item generation. We chose these reports because they focus on threats to future food supply worldwide. Crucially, we assume that threats to the global food supply are necessary for AFS to occur. Whereas threat is not the subject of our study, AFS would not emerge without factors threatening the availability and access to food resources. This process yielded 32 items. Next, we recruited ten native English speakers through Prolific. Their task was to read the AFS definition and propose three items that measure this construct. After removing the items deemed irrelevant and those that depicted unlikely situations in developed countries (e.g., "mass starvation" or "availability of grocery stores"), we added 16 items to the pool. In total, both steps generated 48 items. We did not develop negatively worded items, as such items may bias the results (Podsakoff et al., 2003). Finally, the items were analyzed with the "Hemingway Editor" software (Long, 2020). This software is used for assessing text difficulty, and it indicated that U.S. pupils in the second grade should be able to understand our item pool.

\section{Study 1b: Face validity}

We conducted a face validity study on a target sample to increase the chance that the items were deemed relevant to the construct of interest (Haynes et al., 1995). This study also aimed to reduce the pool of items to those that were the most relevant for measuring AFS.

\section{Participants and procedure}

We recruited 26 native English speakers through Prolific. A similar sample sizes are used in face validity studies involving non-experts recruited online (e.g., Umanath \& 
Coane, 2020). The participants first accepted a consent form and read a definition of AFS. Then, they answered three questions regarding each item ("How relevant is each statement to anticipated food scarcity?"; "How easy is the statement below to understand?"; and "Is the statement below grammatically correct?"). They gauged each item's relevance to AFS on a four-point scale ranging from $0=$ Not at all relevant to $3=$ Very relevant. The participants evaluated how easy the items were to understand. We were not interested in the item difficulty index, which is commonly used in psychometrics, but rather in the participants' impressions of how much effort it took to comprehend the statements. Hence, the participants responded on a five-point scale ranging from $0=$ Very difficult to $4=$ Very easy.

Finally, the participants had to state whether the items were grammatically correct on a binary scale, where $0=N_{o}$ and $1=Y e s$. We randomized the order of the items. In the end, the participants were provided with a blank space in which they could suggest improvements. The study ended with the participants providing their demographic information.

\section{Results and discussion}

We calculated the intraclass correlation coefficient (ICC) (Shrout \& Fleiss, 1979) to assess the agreement between the raters. The intraclass correlation coefficient was obtained by computing a mean-rating, consistency, two-way mixed-effects model (ICC [3,k]) with 26 raters across 48 items measuring the perceived relevance to the AFS. The results $(\mathrm{ICC}=$ $.79,95 \% \mathrm{CI}=[.71, .86])$ suggested moderate-to-good reliability (Koo \& Li, 2016). We removed the items situated below the median scores in the task, where the participants evaluated the relevance of the items to the AFS. This process yielded a 22-item pool. Items that were found to be difficult or grammatically incorrect were reworded.

\section{Study 1c: Pretesting}

We conducted a pretest study to primarily examine the performance of the 22-item pool and to further reword the worst-performing items before conducting the main study. 


\section{Participants and procedure}

A sample of 52 native English speakers was recruited through Prolific. The participants first read and completed a consent form. Then, they read the instructions ("The statements below concern food security by $2050^{1}$. Indicate how strongly you disagree (1) or agree (7) with each statement") and stated how much they agreed with each item. We randomized the order of the items. After filling out the scale, the participants were provided with a blank space in which they could suggest improvements. Finally, they provided their demographic information.

We asked participants about food security rather than food scarcity in instructions because studies on sentence comprehension show that positive sentences are generally easier to understand (e.g., Sherman, 1973). Moreover, people infer the effort needed to complete the task from the difficulty of its instructions, and they are willing to engage less in tasks deemed difficult (Song \& Schwarz, 2008). The word "scarcity" receives 50 times fewer hits in the Google search engine than the word "security." Thus, we deemed the latter term as more straightforward to understand due to its more frequent use. Hence, we instructed participants to think about "food security" to facilitate comprehension and effort put into the task.

\section{Results and discussion}

We first visually inspected the data. Most of the items, with a few exemptions, produced a full range of responses. Skewness and kurtosis outcomes indicated a normal distribution of responses. However, item 5 ("Groceries will be more expensive") produced highly skewed responses (skewness $=-1.65$; kurtosis $=3.6$ ). Indeed, the statement in this item represents a truism; i.e., due to inflation, it is rational to expect all products to become more expensive over time. This item's mean and standard deviation also suggested a ceiling effect $(M=5.81, S D=1.24)$. Therefore, item 5 was flagged for further inspection

\footnotetext{
${ }^{1}$ In Study 4, we asked participants to think about food security in the future and found that changing instructions had no significant effect on the scale's psychometric properties and mean scores.
} 
and rewording. The correlation matrix indicated that some items correlated poorly with the others. Thus, we computed corrected item-scale correlations (each item was correlated with the full scale except with itself). The mean item-scale correlation was .395. Two items did not meet the minimum item-scale correlation of .3 (Boateng et al., 2018); thus, item 3 (item-scale correlation $=.271)$ and item 21 (item-scale correlation $=.200)$ were flagged for further inspection and rewording. Due to low discrimination and potential ceiling effects, we decided to reword seven items. These items were truisms in most cases (for instance, "Some foods will be more expensive"); thus, most of the respondents strongly agreed with them. Based on this process, we then made the items discussed above more specific (for example, "Food prices will rise faster than wages"). No item was removed from the pool after the pretest study; hence, the 22-item AFSS was psychometrically validated in Study 2.

\section{Study 2: Psychometric validation}

Study 2 was conducted to refine and shorten the 22-item pool and to evaluate its psychometric properties. Study 2 left nine items in the pool for further inspection in Study $3 a$.

\section{Participants and procedure}

We recruited 303 native English speakers through Prolific. The participants were mostly from the United States and the United Kingdom. Sixteen participants failed the first attention check question (instructional manipulation check) and were thus removed from analysis; three participants failed the second attention check question (a question regarding the number of siblings; two-digit answers to this question were considered a failure) and were therefore removed from further analysis. This process yielded a final sample of 284 participants (178 women, $M_{a g e}=33.8, S D=12.7$ ).

The participants first read and accepted a consent form. Then, they read the instructions ("The statements below concern food security by 2050. Indicate how strongly you disagree (1) or agree (7) with each statement.") and stated how much they agreed with each item. We randomized the order of the items. Apart from filling out the scale items, 
the participants responded to two attention checks and provided their demographic information.

\section{Results and discussion}

We performed a Mokken scale analysis. The 22-item scale homogeneity $(H=.461$, $S E=.026$ ) indicated a moderate scale (Mokken, 1971; Van der Ark, 2007, 2012). We used the automated item selection procedure algorithm to see how the items performed at increasing homogeneity thresholds. The items that fell below the threshold of .55 were removed from further analyses. Although this threshold was above the recommended minimum of .3 (Hemker et al., 1995), we decided to increase it to achieve a satisfactory scale length. This procedure removed 11 items from the initial pool. Items 12 and 22 violated the invariant item ordering (critical values over 80) (Van Schuur, 2003), so they were removed from subsequent analyses, leaving nine items for further inspection. At this stage, none of the items showed the $H$ coefficient lower than .599 or violated the monotonicity, invariant item ordering, or local independence. Thus, the 9-item solution fulfilled the criteria for the double monotonicity model, and its homogeneity level $(H=$ $.656, S E=.025)$ indicated a strong and unidimensional scale (Mokken, 1971; Van der Ark, 2007, 2012).

We explored the unidimensional model fit with a confirmatory factor analysis using the "lavaan" package (Rosseel et al., 2012). The model fit was good, with the following outcomes: chi-square $(27)=67.515, p<.001$, comparative fit index $(\mathrm{CFI})=.978$, Tucker-Lewis index $(\mathrm{TLI})=.971$, root mean squared error of approximation $(\mathrm{RMSEA})=$ $.073,90 \% \mathrm{CI}=[.051, .095]$, and standardized root mean square residual $(\mathrm{SRMR})=.027$.

The 9-item AFSS had excellent reliability $(\alpha=.94)$. The mean corrected inter-item correlation was .63. The scale means $(M=4.39$, range 4.07 to 4.63$)$ and the standard deviations $(S D=1.34)$ were close to the median, indicating that the scale scores showed no ceiling or floor effects. Scale skewness (-0.31) and kurtosis (-0.46), paired with a visual inspection of the histograms, indicated a normal distribution of the responses. 


\section{Study 3a: Further scale refinement, convergent and discriminant validity}

We conducted Study 3a to further the 9-item AFSS further and to test its convergent and discriminant validity. This procedure yielded the final AFSS scale consisting of 8 items.

\section{Participants and procedure}

We recruited 212 native English speakers through Prolific. Similar to Study 2, the participants were mostly from the United States and the United Kingdom. One participant failed an attention check, which asked about the name of the prime minister or president located in the same country as the participant. This process yielded a final sample of 211 participants (148 women, $M_{\text {age }}=32.3, S D=12.3$ ).

The procedure and instructions to the AFSS were similar to those used in Study 2. Before filling out each questionnaire, we provided separate instructions to ensure that the participants noticed the changing response formats throughout the study (in some cases, the highest agreement rating was the leftmost response, whereas in other cases, it was the rightmost response).

\section{Measures}

Aside from the AFSS, the participants completed the questionnaires described below. We purposely avoided using the popular USDA Food Security Survey Module in this validation study, as this tool primarily captures the lack of financial resources needed to obtain enough food; thus, it may not cover the whole spectrum of factors contributing to food security (Ashby et al., 2016).

Food Insecurity Experience Scale (FIES). To assess the discriminant validity of the AFSS, we used the Food Insecurity Experience Scale developed by the Food and Agriculture Organization of the United Nations (FAO, 2014). The advantage of this experience-based measure is that it captures not only physical food unavailability but also the subjective experience of food insecurity. Specifically, one question asks respondents to state whether they are worried about food sufficiency (Saint Ville et al., 2019). The 
participants responded to each of eight statements on a binary scale, where $0=N_{o}$ and 1 $=$ Yes. Higher scores correspond to higher levels of food insecurity.

Radimer/Cornell Measure of Hunger and Food Insecurity. Another scale that we applied to assess the discriminant validity was the Radimer/Cornell Measure of Hunger and Food Insecurity (Radimer et al., 1990). This tool has four items that encompass worry about food resources in various situations. Furthermore, it allows respondents to state how often such events happen in their lives. The participants responded to each of eight statements on a scale ranging from $1=\mathrm{No}$, never to $5=$ Yes, always. Higher scores correspond to higher levels of food insecurity.

Mini-K. The life-history strategy was measured with the Mini-K, which is a short, 20-item version of the Arizona Life History Battery (Figueredo et al., 2006). According to theory (Hill \& Kaplan, 1999), humans adopt either a fast or slow life history strategy as a response to the low or high availability of resources in their environment to solve trade-offs between somatic effort (saving energy for growth and survival) and reproductive effort (investing energy in reproduction). Life-history strategies can determine variations in human behaviors such as risk-taking, temporal orientation, or proneness to approach temptations. Consequently, studies have found that people who grow up in areas of resource deprivation (environments facilitating fast life-history strategies) are more impulsive and risk-taking, and they approach temptations more quickly than other individuals (Griskevicius et al., 2013). We hypothesized AFS to be positively related to fast life-history strategies, because a shortage of resources favors fast over slow life-history strategy (Figueredo et al., 2006; Griskevicius et al., 2013). The participants responded on a seven-point scale ranging from $-3=$ Disagree strongly to $3=$ Agree strongly. Higher scores indicate a slower life-history strategy.

HEXACO-60 Personality Inventory. We measured one personality dimension from the HEXACO-60 short personality inventory (Ashton \& Lee, 2009). Considering that environmental threats have been linked to higher anxiety levels (Twenge, 2000), we 
hypothesized that more emotional people perceive future food resources as being scarcer compared to the perception of less emotional people. The participants responded to the 10-item measure of emotionality on a five-point scale ranging from $1=$ Strongly disagree to $5=$ Strongly agree. Higher scores correspond to higher levels of emotionality.

Generalized Sense of Power Scale. The 8-item Generalized Sense of Power Scale (Anderson \& Galinsky, 2006) was used to test the prediction that people who believe that they can wield power over others perceive future food resources as more secure as such people may see more ways of obtaining food and other resources than those who have a lower sense of power. Participants responded on a seven-point scale ranging from $1=$ Disagree strongly to $7=$ Agree strongly. Higher scores represent a higher sense of power.

Locus of Control Scale. The 29-item Locus of Control Scale (Rotter, 1966) measures the external versus internal locus of control among participants. Locus of control determines whether people perceive their actions as having an impact on the outcomes from many facets of their daily life. We hypothesized that people with a more external locus of control would score higher on the AFSS, as these individuals believe they do not have control over events such as agricultural turmoil. There are 29 items in total, with two alternatives. The participants had to choose one of the two alternative statements that they agreed with the most. High scores correspond to an external locus of control.

\section{Results and discussion}

One item lowered the scale's alpha value, and its coefficient of variation was the highest; thus, this item was flagged for further inspection. This item also had a much lower item discrimination coefficient than the rest of the items (these indicators combined suggested that this item could have failed to differentiate well between the participants at different levels of the latent variable).

We performed a Mokken scale analysis, as described in Study 2. The 9-item AFSS homogeneity coefficient $(H=.742, S E=.026)$ indicated a strong scale (Mokken, 1971; Van der Ark, 2007, 2012). The automated item selection procedure algorithm suggested 
that the scale was unidimensional; we did not find any group of items "departing" at a certain homogeneity level. No item violated the monotonicity or local independence. One item exceeded the suggested critical value of 80 for invariant item ordering (Van Schuur, 2003), but this violation was not significant. Therefore, the 9-item scale was found to meet the criteria for the double monotonicity model (Mokken, 1971). The item flagged for potential removal in the earlier step contributed to the lowest homogeneity coefficient and violated monotonicity at lower rest score group sizes (Van der Ark, 2007). Thus, this item was removed from further analysis, yielding the 8-item AFSS.

We explored the unidimensional model fit with a confirmatory factor analysis. The model fit was acceptable, with outcomes as follows: chi-square $(20)=55.602, p<.001$, comparative fit index $(\mathrm{CFI})=.980$, Tucker-Lewis index $(\mathrm{TLI})=.972$, root mean squared error of approximation $(\mathrm{RMSEA})=.092,90 \% \mathrm{CI}=[.064, .121]$, and standardized root mean square residual $(\mathrm{SRMR})=.019$.

Similar to Study 2 , the 8 -item AFSS had excellent reliability $(\alpha=.96)$. The mean corrected inter-item correlation was .76. The 8-item mean $(M=4.60$, range 4.34 to 4.82$)$ and standard deviation $(S D=1.47)$ indicated a lack of ceiling or floor effects (see Table 3 for the final items included in the scale). Scale skewness (-0.53) and kurtosis (-0.29), as well as a visual inspection of the histograms, indicated a normal distribution of the responses.

The 8-item AFSS showed low but statistically significant correlations with the Food Insecurity Experience Scale (Kendall's $\tau=.12, p=.017$ ) and the Radimer/Cornell Measure of Hunger and Food Insecurity (Kendall's $\tau=.14, p=.006$ ). These results provide evidence that anticipated food scarcity is a different construct than the food insecurity studied in the existing literature that refers to retrospective/actual experiences of food unavailability. Interestingly, the AFSS did not correlate with the Mini-K ( $r=$ $-0.01, p=.867)$, suggesting that life-history strategies and anticipated food scarcity are unrelated constructs. As predicted, the AFSS correlated positively with the Locus of Control Scale $(r=.21, p=.002)$ and with the emotionality dimension of the HEXACO 
Personality Inventory $(r=.19, p=.005)$. These outcomes suggest that people with an external locus of control and those who are more emotional perceive future food resources as being scarcer. Finally, we found a negative correlation between the AFSS and the Generalized Sense of Power Scale $(r=-0.16, p=.018)$. This result is in line with our prediction that people who believe that they can convince others to do what they want to perceive future food resources as more secure, perhaps because they are more capable of finding ways to access food and other resources. Table 3 provides a summary of the correlations between the 8-item AFSS and the other included scales.

Studies 2 and 3a jointly confirmed the good psychometric properties of the 8-item AFSS. Although we found significant correlations according to our predictions, the AFSS was only correlated weakly with the scales mentioned above. This means that anticipated food scarcity is a new construct that warrants future investigation. To further support this notion, we performed additional analyses where we controlled for the two existing food insecurity measures that we described above. These analyzes revealed that the AFSS was positively related to emotionality $(p=.010)$. However, both the Food Insecurity Experience Scale and the Radimer/Cornell Measure of Hunger and Food Insecurity were not related to this dimension (both $p s>.549)$. Similarly, only the AFSS $(p=.003)$ was related to locus of control, but the remaining two scales were not (both $p s>.401$ ). Further, both the AFSS and the Radimer/Cornell Measure of Hunger and Food Insecurity were marginally related to the Generalized Sense of Power Scale (both $p s<.082$ ), as opposed to the Food Insecurity Experience Scale $(p=.771)$. Interestingly, the existing tools were related to life-history strategy (both $p s<.079)$, but the AFSS was not $(p=.712)$.

The low correlations between the AFSS and scales described in Table 3 are unsurprising given that AFS is a perception. In contrast, the scales we compared it against reflect personality traits and past experiences. It is plausible that current perceptions of future food resources as scarce are affected by past experiences of food unavailability; hence, the FIES and Radimer/Cornell Measure of Hunger and Food Insecurity are weakly 
but positively associated with scores on the AFSS. Considering that emotionality is positively related to neuroticism, and multiple items capturing this trait focus on anxiety and fearfulness (Ashton \& Lee, 2009), it is justified to expect that AFS, to some extent, captures affective reactions (e.g., anxiety triggered by cues related to food scarcity).

Indeed, Studies 3a and 5 partially supported this notion. Study 3a provided evidence that people who believe that they have little influence over what happens in the world around them (i.e., having an external locus of control and a low sense of power) score higher on the AFSS. These results align with our definition of this construct and the items capturing it. People who believe they can wield power over others and those who have an internal locus of control likely also think that they can obtain access to food resources despite these becoming scarcer. This access depends on their decisions rather than on external circumstances. In sum, Study 3 a showed that the AFSS is a construct distinct from the existing food insecurity measures.

\section{Table 2}

The final set of items included in the AFSS

AFS $1 \quad$ Food shortages will occur more frequently

AFS 2 Food resources will become increasingly scarce

AFS 3 There will be less food available

AFS 4 Food availability will be more uncertain

AFS 5 More people will be hungry

AFS 6 The demand for food will be higher than the supply

AFS 7 There may not be enough food for everyone

AFS $8 \quad$ People will compete for food resources

\section{Study 3b: Additional tests of convergent and discriminant validity}

Study 3a shows that AFS is a distinct construct to food insecurity commonly treated in the literature as the physical experience of food insufficiency. However, Study 3a 
did not investigate if AFS is not a ramification of general anxiety or other emotional states. Thus, Study 3b tests this possibility. Additionally, we investigated whether AFSS predicts social attitudes, namely - support for universal basic income and an attitude toward welfare recipients.

\section{Participants and procedure}

We recruited 140 participants from the United States through Prolific (68 women, $\left.M_{a g e}=35, S D=12.8\right)$. The data were collected in connection to another research project, which examined the influence of seasonal cues on food preferences.

After accepting an informed consent form, participants provided their Prolific IDs and watched a 50-second video showing either a winter or summer forest walk from the first-person perspective. These videos were devoid of emotionally-laden stimuli. Then, they filled out scales described in measures. The study concluded by providing demographic data and answering an attention check question regarding the video's contents.

\section{Measures}

We measured AFS, positive and negative affect, as well as anxiety. Moreover, we investigated if the AFSS was linked to gradients of prosocial behavior.

Positive and Negative Affect Schedule (PANAS). Positive and negative affect was captured on a 20-item (10 for positive and 10 for negative affect) Positive and Negative Affect Schedule (PANAS) (Watson et al., 1988), with responses ranging from $1=$ very slightly or not at all to $5=$ extremely.

Short form of the State-Trait Anxiety Inventory (STAI). We measures anxiety using a six-item short-form of the Spielberger State-Trait Anxiety Inventory (Marteau \& Bekker, 1992), with responses ranging from $1=$ Not at all to $4=$ Very much .

\section{Prosociality: support for Universal Basic Income and attitudes toward} welfare recipients. Several theories pose that food scarcity and social behavior may be related. The hunger hypothesis (Nettle, 2017) postulates that people of lower socioeconomic position are more impulsive, irritable, aggressive, and experience more 
anxiety than people of higher socioeconomic classes partly due to food insufficiency and food insecurity prevalent in their environments. According to the conservation of resources theory (Hobfoll, 1989), people facing threats (e.g., food scarcity) strive to keep resources to themselves rather than to share these with others. Hence, as anticipating food scarcity may prompt people to maximize their own (vs. societal) welfare, we predict that AFS will be positively related to the support for Universal Basic Income and negatively related to attitudes toward welfare recipients.

Participants read a definition of the Universal Basic Income and stated their support for this idea ("How bad or good an idea would it be to introduce a Universal Basic Income in your country?") on a sliding scale ranging from $0=$ Bad idea to $100=$ Good idea (Nettle et al., 2020).

They voiced their attitudes toward welfare recipients by agreeing with the two statements ("Too many get social welfare without needing it"; "Many of the unemployed don't really want to find work.") on a scale ranging from $1=$ disagree to $5=$ agree (Petersen et al., 2014).

\section{Results and discussion}

The AFSS was unrelated to positive affect $(r=.04, p=.634)$, but correlated with negative affect $(r=.21, p=.012)$ and anxiety $(r=.23, p=.007)$. In line with our predictions, the AFSS was positively related to support for Universal Basic Income $(r=$ $.19, p=.027)$. But, we found the construct unrelated to attitudes toward welfare recipients $(r=.03, p=.723)$.

Because participants have watched a short video clip before filling out the scales, we tested whether these videos affected subsequent responses. We performed a multivariate analysis of variance (MANOVA) with all the dependent variables and the two predictors: experimental condition (watching summer or winter forest walk) and participant sex. Neither participant sex $(p>.462)$, nor experimental condition $(p>.376)$ was related to any dependent measure. 
These findings (see Table 3) suggest that AFS is weakly related to negative affect and anxiety, and it is unrelated to positive affect. Thus, the construct cannot be deemed as a direct ramification of emotional states. Although AFS was unrelated to attitudes toward welfare recipients, we have provided preliminary evidence that it can be predictive of other social behavior gradients - support for Universal Basic Income. Therefore, it is plausible that AFS can deepen the understanding of complex social interaction and resource distribution.

\section{Table 3}

Correlations between AFSS scores and scores on the other scales

\begin{tabular}{llll}
\hline Scale & Statistic & Coefficient & $p$-value \\
\hline FIES & Kendall's $\tau$ & .12 & $\mathbf{. 0 1 7}$ \\
Radimer/Cornell's & Kendall's $\tau$ & .14 & $\mathbf{. 0 0 6}$ \\
Mini-K & Pearson's $r$ & -0.01 & .867 \\
HEXACO-60 (emotionality) & Pearson's $r$ & .19 & $\mathbf{. 0 0 5}$ \\
Generalized Sense of Power Scale & Pearson's $r$ & -0.16 & $\mathbf{. 0 1 8}$ \\
Locus of Control Scale & Pearson's $r$ & .21 & $\mathbf{. 0 0 2}$ \\
PANAS (positive affect) & Pearson's $r$ & .04 & .634 \\
PANAS (negative affect) & Pearson's $r$ & .21 & $\mathbf{. 0 1 2}$ \\
STAI & Pearson's $r$ & .23 & $\mathbf{. 0 0 7}$ \\
Support for Universal Basic Income & Pearson's $r$ & .19 & $\mathbf{. 0 2 7}$ \\
Attitude toward welfare recipients & Pearson's $r$ & .03 & .723 \\
\hline
\end{tabular}

Note. We report the parametric correlation coefficient (Pearson's $r$ ) whenever the responses approach normal distributions and data are continuous. The nonparametric correlation coefficient (Kendall's $\tau$ ) is reported for highly skewed and ordinal data. 


\section{Study 4: Criterion validity}

We conducted Study 4 to test whether the AFSS can predict food preferences. In the earlier studies, the instructions for participants mention a specific and distant time frame, namely - the year 2050. Thus, we wanted to check whether altering instructions to more generic yields a different pattern of responses. Finally, Study 4 aimed to confirm the psychometric properties with a higher sample size.

\section{Participants and procedure}

Data for Study 4 were collected simultaneously with data for another preregistered research (https://osf.io/efyu3). We recruited 415 participants from the United States (204 women, $\left.M_{a g e}=36.9, S D=13\right)$ through Prolific.

Participants first read and accepted a consent form. Then, they provided their demographic data, filled out the 8-item AFS Scale ("The statements below concern food security in the future. Indicate how strongly you disagree (1) or agree (7) with each statement"), and were randomly assigned to one of the two conditions. After watching their given video, they estimated the caloric content and stated their preferences for 30 foods. The order of these two tasks (estimating calories and stating preferences) was randomized. Likewise, the order of the 30 food images within each task was randomized. Finally, they answered an attention check question about the season present in the video they watched.

\section{Measures}

We used food images from Folwarczny et al. (2021). This set of 30 simple foods and complex dishes has been pretested on a sample of certified nutritionists and athletes who measure foods' caloric content daily. The set covers a broad spectrum in calorie estimates $\left(M_{\text {calories }}=367, S D=122\right.$, range $\left.=166-711\right)$ and has been applied in experimental research (Folwarczny et al., 2021).

To evaluate the calorie content of foods ("How many calories does this food contain? [the serving you see]"), participants used a sliding scale ranging from 0 to 1000 calories (kcal), with 1 point intervals. We have considered the experts' estimation of the calorie 
content of foods when creating this response format.

Participants answered questions regarding their preferences toward foods ("Would you eat this food now?") on a sliding scale ranging from -1 (Definitely not) to 1 (Definitely yes), with .01 point intervals.

\section{Results: Scale psychometric properties}

We performed a Mokken scale analysis, as described in Studies 2-3a. The 8-item AFSS homogeneity coefficient $(H=.823, S E=.015)$ indicated a strong scale (Mokken, 1971; Van der Ark, 2007, 2012). The automated item selection procedure algorithm suggested that the scale was unidimensional; we did not find any group of items "departing" at a certain homogeneity level. No item significantly violated the monotonicity, local independence, or invariant item ordering (Van Schuur, 2003). Therefore, the 8-item scale was found to meet the criteria for the double monotonicity model (Mokken, 1971).

We explored the unidimensional model fit with a confirmatory factor analysis. The model fit was acceptable, with outcomes as follows: chi-square $(20)=105.186, p<.001$, comparative fit index $(\mathrm{CFI})=.979$, Tucker-Lewis index $(\mathrm{TLI})=.971$, root mean squared error of approximation $(\mathrm{RMSEA})=.101,90 \% \mathrm{CI}=[.083, .121]$, and standardized root mean square residual $(\mathrm{SRMR})=.017$.

Similar to Studies $2-3 \mathrm{a}$, the 8 -item AFSS had excellent reliability $(\alpha=.97)$. The mean corrected inter-item correlation was .76. The 8-item mean $(M=4.38$, range 4.18 to 4.64) and standard deviation $(S D=1.49)$ indicated a lack of ceiling or floor effects. Scale skewness (-0.32) and kurtosis (-0.66), as well as a visual inspection of the histograms, indicated a normal distribution of the responses.

\section{Results: Criterion validity}

We created the food preference index $(\alpha=.88)$ by averaging responses to 30 questions about a desire to consume each food. Likewise, we created an index of calorie estimates $(\alpha=.94)$ by averaging 30 foods' caloric content judgments.

We fit a simple linear regression model with food preference index as a dependent 
variable and AFSS index as a predictor. This analysis revealed that AFSS was predictive of overall food preferences, $b=.031, S E=.010, t=3.04, p=.003$. These results remained significant $(p=.002)$ even when controlling for participant sex, age, and experimental condition.

A similar analysis with calorie estimates index as a dependent variable suggested that AFSS was predictive of calorie judgements, $b=7.920, S E=3.617, t=2.19, p=.029$. Again, the results were significant $(p=.033)$ even when adding covariates to the model.

We performed a simple slope analysis to investigate whether AFSS predicts food preferences in both conditions. This analysis found a slope of AFSS index significant among participants exposed to videos showing winter $(p=.004)$, but not among their peers exposed to videos showing summer $(p=.133)$. Figure 1 shows these differences.

\section{Figure 1}

Differences between the groups in AFS scores

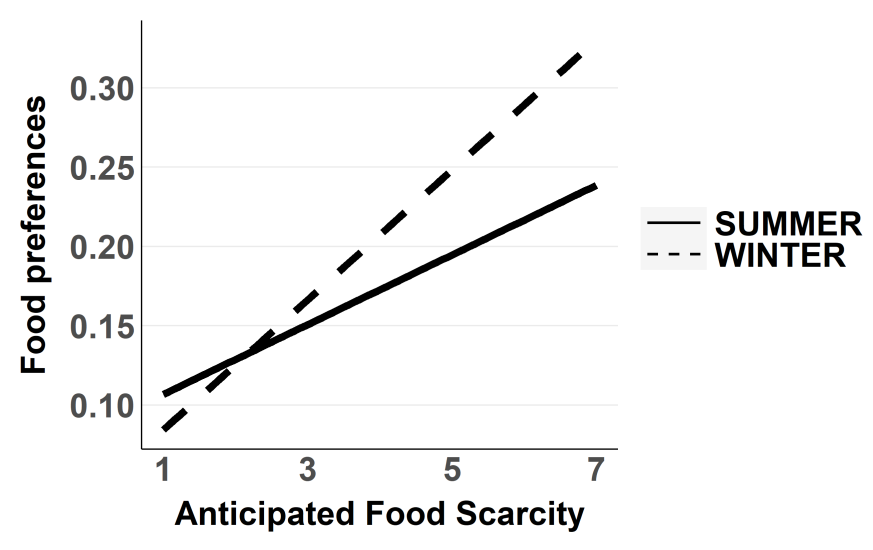

Considering that AFS is positively related to overall food preference and calorie estimates, it is plausible that foods' perceived caloric content drives this increase in food preferences. We tested this notion by performing a mediation analysis with the "mediation" package for R (Tingley et al., 2014). Nonparametric bootstrap confidence intervals were obtained with 50000 simulations. We report the standardized coefficients below.

As Figure 2 indicates, the relationship between AFS and food preferences was 
mediated by perceived caloric content of foods $(\mathrm{ACME}=.02, p=.031,95 \% \mathrm{CI}=[.00$, $.05] ; \mathrm{ADE}=.13, p=.017,95 \% \mathrm{CI}=[.02, .23]$; Total Effect $=.15, p=.005,95 \% \mathrm{CI}=$ $[.04, .25]$; Prop. Mediated $=.14, p=.036,95 \% \mathrm{CI}=[.01,0.53]$.

\section{Figure 2}

Mediation model with standardized coefficients

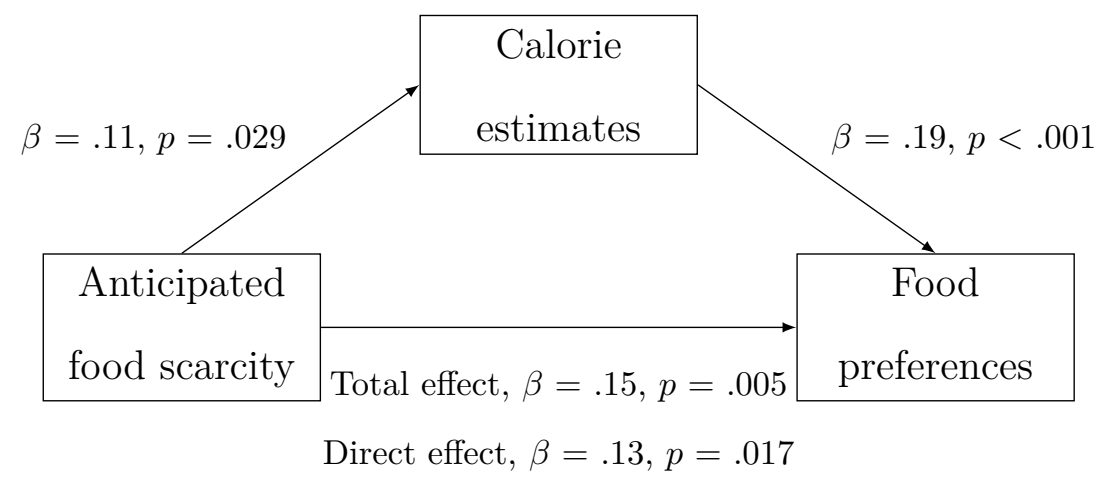

\section{Discussion}

Study 4 confirmed the good psychometric properties of the AFSS, thus mirroring findings from Studies 2 and 3a. Additionally, the study revealed that the AFSS was positively related to general food preferences and calorie judgments. Calorie judgments mediated the relationship between AFS and food preferences.

A simple slope analysis found that the AFSS index was positively related to food preferences among participants who watched videos showing winter but not summer. Winter is - arguably - a genuine cue to food scarcity. Indeed, from noncomplex plants to polar bears, organisms respond to seasonal changes in the food web (e.g., lowered food availability in the winter) by reducing energy expenditure and increasing fat reserves (Blix, 2016; Humphries et al., 2017; Lima, 1986).

\section{Study 5: Sensitivity to food scarcity cues}

Anticipated food scarcity — unlike food insecurity as it is commonly defined in the current literature - is a perception and thus should, to a certain extent, be under the short-term influence of external factors, such as exposure to news reports. Study 5 
investigated if exposing participants to pictorial and textual cues to food scarcity indeed increases their AFS.

\section{Participants and procedure}

We decided in advance to recruit at least 40 participants per cell. A stochastic power simulation (Bolker, 2007) revealed that this sample size would provide a power of .8 to detect a significant difference between two groups, assuming a medium-to-large effect size (Cohen's $d=.65$ ). We recruited 175 native English speakers (111 women, $M_{\text {age }}=34.2$, $S D=12.1)$ through Prolific. Again, the participants were mostly from the United States and the United Kingdom. No one failed the attention check ("Who is the prime minister or president of your country?").

The participants first read and accepted a consent form. Then, they were randomly assigned to one of four groups, thereby causing the study to adopt a single factor between-subjects design. Depending on their assigned condition, the participants were either told to read an article or watch a video and memorize critical facts from the content. The participants in the two experimental groups were exposed to food scarcity content, and those in the two control groups were exposed to stimuli unrelated to food. More specifically, the participants in the first control group read an article entitled "Why Are Swiss Watches So Expensive?," whereas those in the second control group watched a 67-second-long subtitled video entitled, "Airlines don't have to leave the middle seats on flights empty anymore." The participants who were assigned to the two experimental groups had to read an article entitled "Food Insecurity Remains Long Term Problem," or, alternatively, watch a 66-second-long subtitled video entitled "Will COVID-19 lead to a global food crisis?" All of the articles and videos were made available through this project's OSF webpage. After reading the article or watching the video, the participants completed the AFSS (we used the same instructions as these reported in Studies 1c-3a) and the short, 10-item version of the Positive and Negative Affect Schedule (Thompson, 2007). 


\section{Measures}

Both articles were approximately 500 words long and can be accessed through this project's OSF webpage. These articles were downloaded from EzineArticles.com. The control group read an article about the factors underlying the high cost of Swiss watches, whereas the experimental group read about the increasing problem of food insecurity worldwide. The stimuli videos came from YouTube.com. The control group watched a 67-second-long video about COVID-19 preventive measures being lifted in the airline industry (this video did not cover any food-related topics). The experimental group watched a 66-second-long video about a looming famine in many parts of the world being triggered by the pandemic.

Positive and negative affect elicited by the stimuli, as measured through the 10-item short version of the Positive and Negative Affect Schedule (I-PANAS-SF) (Thompson, 2007), were included to control for potential affective differences between the participants across the conditions. The I-PANAS-SF contains two subscales that measure positive and negative affect, with each subscale having five items. The order of items was randomized, and the participants responded on a five-point scale ranging from $1=$ Very slightly or Not at all to $5=$ Extremely. High scores on each subscale are indicators of high levels of positive or negative affect.

\section{Results and discussion}

We averaged the scores from the AFSS $(\alpha=.95)$, positive $(\alpha=.76)$, and negative affect scales $(\alpha=.86)$ (Thompson, 2007). Then, we tested whether the control and experimental groups differed in their AFS levels. Neither of the two control groups $(p>$ $.208)$ nor either of the two experimental groups $(p>.542)$ reported different AFS levels. Thus, both the control and experimental groups were combined to facilitate parsimonious analyses (cf. Griskevicius et al., 2009; Otterbring \& Sela, 2020).

Notably, the experimental groups $(M=5.54, S D=1.11)$ reported higher levels of anticipated food scarcity than did the control groups $(M=4.73, S D=1.44), t(173)=$ 
-4.13, $p<.001$, Cohen's $d=.63$. The experimental $(M=2.77, S D=0.76)$ and control $(M=2.70, S D=.80)$ groups reported the same levels of positive affect, $t<1$. However, the experimental groups $(M=1.97, S D=.80)$ reported higher levels of negative affect than did the control groups $(M=1.46, S D=.74), t(173)=-4.36, p<.001$, Cohen's $d=$ .66. To test the robustness of our findings and to ensure that food scarcity exposure drove the increase in AFS scores despite the differences in self-reported negative affect, we conducted a further analysis in which we controlled for the participants' negative affect. Specifically, we performed an analysis of covariance (ANCOVA) with a participant group as the independent variable, negative affect as the covariate, and AFS scores as the dependent variable. The covariate - negative affect - was significantly related to AFS scores, $F(1,172)=19.73, p<.001$. Importantly, there was also a significant group effect on AFS scores after controlling for negative affect, $F(1,172)=7.44, p=.007$. These results suggest that experimentally manipulated food scarcity, in terms of both visual and textual food scarcity content, increases AFS scores (see Figure 3).

\section{Figure 3}

Differences between the groups in AFS scores

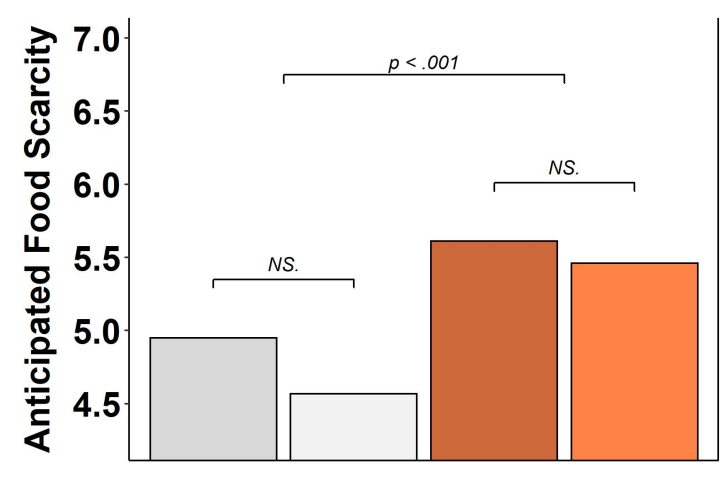

\section{GROUP \\ CONTROL (TEXT) \\ CONTROL (VIDEO) \\ EXPERIMENTAL (TEXT) \\ EXPERIMENTAL (VIDEO)}




\section{General discussion}

The current research examined the psychometric properties of the newly developed 8-item Anticipated Food Scarcity Scale (AFSS), which can be used to measure people's perceptions of food resources becoming less available. Anticipated food scarcity (AFS), as it is studied herein, is a new psychological construct that can contribute to the understanding of factors underlying food hoarding behaviors, impulsivity, or increased preferences toward energy-dense foods when consumers are exposed to food scarcity (cf. Folwarczny et al., 2021; Nettle et al., 2017). In contrast to the existing measures-such as HFIAS (Coates et al., 2007) — that focus on past experiences of food insecurity threatening relatively few people living in developed countries, the AFSS captures future-related perceptions among the general population.

The 8-item AFSS forms a strong, unidimensional scale that meets the criteria for the double monotonicity model (Van der Ark, 2007, 2012). The low correlations between the AFSS and Food Insecurity Experience Scale (FAO, 2014), as well as the Radimer/Cornell Measure of Hunger and Food Insecurity (Radimer et al., 1990), indicate that AFS and food insecurity, as treated in the current literature, are qualitatively distinct but presumably interrelated constructs. We also found that people with an external locus of control (Rotter, 1966), a lower sense of power (Anderson \& Galinsky, 2006), and higher levels of emotionality (Ashton \& Lee, 2009) perceive future food resources as being scarcer. Life-history strategy (Figueredo et al., 2006) and positive affect (Watson et al., 1988) were unrelated to AFS scores. Although the AFSS shows positive correlations with negative affect and anxiety (Marteau \& Bekker, 1992; Watson et al., 1988), these correlations were

weak. More importantly, Study 5 shows that people exposed to food scarcity content score higher ion the AFSS, and these results remain significant even when controlling for negative affect (Thompson, 2007). Therefore, it is unlikely that the AFSS captures merely general anxiety or negative affect associated with fear of losing access to food resources. Building on the literature postulating that people facing difficulties keep resources to 
themselves rather than sharing them with others (Hobfoll, 1989), we predicted and found that AFS was positively related to support Universal Basic Income (Nettle et al., 2020). However, the scale was not predictive of attitudes toward welfare recipients (Petersen et al., 2014). Taken together, these findings show that AFS may be linked to prosocial behaviors and food preferences.

The AFSS is sensitive to food scarcity cues. In Study 5, we exposed participants to either an article describing globally rising food scarcity, a video about COVID-19-related food scarcity, or control stimuli. The participants exposed to textual and visual food scarcity content reported higher AFS levels. This outcome suggests that the AFSS can be used in experimental work where researchers may be interested in manipulating AFS.

The results reported above support the evolutionary mismatch hypothesis (Li et al., 2018). A mismatch occurs when our psychological mechanisms, which are largely adapted to savannah-like environments (within which our ancestors resided and adapted for millions of years), interact with evolutionarily novel stimuli from our modern world, thereby producing maladaptive outcomes. As a consequence, our evolved mechanisms are often outdated and no longer help us maximize our adaptiveness in the modern world. The environments that fashioned the adaptations that protected our ancestors against periods of food unavailability are vastly different from the current environments.

Nonetheless, psychological mechanisms did not evolve to distinguish between current and impending threats and those that might occur decades later because such distal threats are largely caused by technological developments that have only taken place in recent years. Thus, the evolutionarily outdated mechanisms may still respond to the same types of threat stimuli in similar ways that are now maladaptive (Li et al., 2018). For instance, recent research suggests that watching climate change-induced food scarcity videos immediately increases preferences toward energy-dense foods (Folwarczny et al., 2021). Consistently, Study 5 revealed an increase in AFS scores after exposure to food scarcity content. Together, these findings provide convergent support for the evolutionary 
mismatch framework.

\section{Limitations and future research}

Although our participants were primarily from developed countries, half of them reported having a college degree, and only $18 \%$ reported being unemployed; it remains uncertain how many of them were the recipients of food stamps or related programs. One study found all parents receiving food parcels worried about food insufficiency (Harvey, 2016). Thus, it is plausible that these participants react to food scarcity differently than other individuals. Therefore, future studies should investigate more diverse populations. Likewise, the mean AFSS scores reported herein may not indicate the magnitude of the latent variable in less-developed countries.

The degree to which people may experience food scarcity differs dramatically between developed and less-developed countries. Due to potential habituation, the latter may not respond to the same manipulations used in Study 5. Thus, we cannot claim that our scale is sensitive to food scarcity cues found in countries where people are frequently threatened by actual hunger. Future research should investigate samples in which the experience of actual food scarcity is prevalent. Furthermore, we used overt cues to food scarcity in Study 5 (i.e., even the titles of the articles and videos used in Study 5 mention food unavailability). It is vital to determine whether the scale is sensitive to more subtle cues to food scarcity, such as reports showing rising global temperatures.

Aside from investigating how severity influences AFS, it would be interesting to test whether the scale is sensitive to the likelihood of food scarcity. For instance, studies could test whether imminent food scarcity yields the same responses as food scarcity that may or may not occur. It is also unknown whether people react similarly to lower levels of food stocks in general as they do to the disappearance of specific foods.

Finally, the AFSS has not been validated with behavioral measures. Study 4 showed that it could predict food preferences and calorie judgments, and Study 5 showed that the scale is sensitive to food scarcity cues. Although these results are promising, the present 
findings do not imply that the scale has been validated comprehensively. More studies should establish the scale's predictive validity by, for instance, testing whether high AFS scores correspond to choosing energy-dense over low-calorie foods in laboratory and field experiments. Likewise, it is vital to investigate whether AFS scores can predict the rate of obesity or overweight in populations of interest.

\section{Conclusions}

Existing measures have been developed primarily to gauge the prevalence of food insufficiency among low-income populations. However, societies worldwide are consistently informed about publicized events in mass media, such as Brexit, climate change, or pandemics, which pose a threat to the food supply chain. As a result, social groups other than low-income populations may also perceive that food resources are becoming scarcer. Although numerous scientific tools exist to measure actual food insecurity (i.e., hunger), none of these tools capture anticipated food scarcity. To address this shortcoming, we developed and psychometrically validated the 8-item AFSS, which was found to be unidimensional and to have good psychometric qualities. Because the scale is sensitive to food scarcity cues, it can be used in experimental research. Its relatively narrow set of items makes it an exceptionally potent tool for use in online surveys, field settings, and lab studies. In sum, the AFSS presents an alternative approach to food scarcity measurement in affluent societies. Consequently, it can help foster novel research on food waste, sustainability, and a multitude of prosocial behaviors.

\section{Acknowledgments}

We would like to thank Alexandra Dima for her support and suggestions on the analytical approach presented in this research. 


\section{References}

Anderson, C., \& Galinsky, A. D. (2006). Power, optimism, and risk-taking. European Journal of Social Psychology, 36(4), 511-536. https://doi.org/https://doi.org/10.1002/ejsp.324

Ashby, S., Kleve, S., McKechnie, R., \& Palermo, C. (2016). Measurement of the dimensions of food insecurity in developed countries: A systematic literature review. Public Health Nutrition, 19(16), 2887-2896. https://doi.org/https://doi.org/10.1017/S1368980016001166

Ashton, M. C., \& Lee, K. (2009). The HEXACO-60: A short measure of the major dimensions of personality. Journal of Personality Assessment, 91(4), 340-345. https://doi.org/10.1080/00223890902935878

Benker, B. (2021). Stockpiling as resilience: Defending and contextualising extra food procurement during lockdown. Appetite, 156, 104981. https://doi.org/10.1016/j.appet.2020.104981

Blix, A. S. (2016). Adaptations to polar life in mammals and birds. Journal of Experimental Biology, 219(8), 1093-1105. https://doi.org/10.1242/jeb.120477

Boateng, G. O., Neilands, T. B., Frongillo, E. A., Melgar-Quiñonez, H. R., \& Young, S. L. (2018). Best practices for developing and validating scales for health, social, and behavioral research: A primer. Frontiers in Public Health, 6, 149. https://doi.org/10.3389/fpubh.2018.00149

Bolker, B. (2007). Stochastic simulation and power analysis. https://ms.mcmaster.ca/ bolker/emdbook/chap5A.pdf

Casalicchio, E. (2020). Panic-buying in UK will return ahead of Brexit transition end, experts say. https://www.politico.eu/article/uk-panic-buying-set-to-return-aheadof-brexit-transition-end/

CDC. (2019). Climate change and public health. https://www.cdc.gov/climateandhealth/effects/food_security.htm 
Coates, J., Swindale, A., \& Bilinsky, P. (2007). Household Food Insecurity Access Scale (HFIAS) for measurement of food access: Indicator guide. https: //www.fantaproject.org/sites/default/files/resources/HFIAS_ENG_v3_Aug07.pdf

Dima, A. L. (2018). Scale validation in applied health research: Tutorial for a 6-step R-based psychometrics protocol. Health Psychology and Behavioral Medicine, 6(1), 136-161. https://doi.org/10.1080/21642850.2018.1472602

FAO. (1996). World Food Summit Plan of Action. http://www.fao.org/3/w3613e/w3613e00.htm

FAO. (2014). Food Insecurity Experience Scale. http://www.fao.org/in-action/voices-of-the-hungry/fies/en/

FAO. (2016). Climate change and food security: Risks and responses. http://www.fao.org/3/a-i5188e.pdf

Figueredo, A. J., Vásquez, G., Brumbach, B. H., Schneider, S. M., Sefcek, J. A., Tal, I. R., Hill, D., Wenner, C. J., \& Jacobs, W. J. (2006). Consilience and life history theory: From genes to brain to reproductive strategy. Developmental Review, 26(2), 243-275. https://doi.org/10.1016/j.dr.2006.02.002

Folwarczny, M., Christensen, J. D., Li, N. P., Sigurdsson, V., \& Otterbring, T. (2021).

Crisis communication, anticipated food insecurity, and food preferences:

Preregistered evidence of the insurance hypothesis. Food Quality and Preference, 91, 104213. https://doi.org/10.1016/j.foodqual.2021.104213

Galanakis, C. M. (2020). The food systems in the era of the coronavirus (COVID-19) pandemic crisis. Foods, 9(4), 523. https://doi.org/10.3390/foods9040523

Garfin, D. R., Silver, R. C., \& Holman, E. A. (2020). The novel coronavirus (COVID-2019) outbreak: Amplification of public health consequences by media exposure. Health Psychology. https://doi.org/10.1037/hea0000875

Griskevicius, V., Ackerman, J. M., Cantú, S. M., Delton, A. W., Robertson, T. E., Simpson, J. A., Thompson, M. E., \& Tybur, J. M. (2013). When the economy 
falters, do people spend or save? Responses to resource scarcity depend on childhood environments. Psychological Science, 24 (2), 197-205. https://doi.org/10.1177/0956797612451471

Griskevicius, V., Cantú, S. M., \& Van Vugt, M. (2012). The evolutionary bases for sustainable behavior: Implications for marketing, policy, and social entrepreneurship. Journal of Public Policy $\&$ Marketing, 31(1), 115-128. https://doi.org/10.1509/jppm.11.040

Griskevicius, V., Goldstein, N. J., Mortensen, C. R., Sundie, J. M., Cialdini, R. B., \& Kenrick, D. T. (2009). Fear and loving in Las Vegas: Evolution, emotion, and persuasion. Journal of Marketing Research, 46 (3), 384-395. https://doi.org/10.1509/jmkr.46.3.384

Hanjra, M. A., \& Qureshi, M. E. (2010). Global water crisis and future food security in an era of climate change. Food Policy, 35(5), 365-377.

https://doi.org/10.1016/j.foodpol.2010.05.006

Harvey, K. (2016). "When I go to bed hungry and sleep, I'm not hungry": Children and parents' experiences of food insecurity. Appetite, 99, 235-244. https://doi.org/10.1016/j.appet.2016.01.004

Haynes, S. N., Richard, D., \& Kubany, E. S. (1995). Content validity in psychological assessment: A functional approach to concepts and methods. Psychological Assessment, 7(3), 238-247. https://doi.org/10.1037/1040-3590.7.3.238

Hemker, B. T., Sijtsma, K., \& Molenaar, I. W. (1995). Selection of unidimensional scales from a multidimensional item bank in the polytomous Mokken IRT model. Applied Psychological Measurement, 19(4), 337-352. https://doi.org/10.1177/014662169501900404

Hill, K., \& Kaplan, H. (1999). Life history traits in humans: Theory and empirical studies. Annual Review of Anthropology, 28(1), 397-430. https://doi.org/10.1146/annurev.anthro.28.1.397 
Hobfoll, S. E. (1989). Conservation of resources: A new attempt at conceptualizing stress. American Psychologist, 44(3), 513-524. https://doi.org/10.1037/0003-066X.44.3.513

Humphries, M. M., Studd, E. K., Menzies, A. K., \& Boutin, S. (2017). To everything there is a season: Summer-to-winter food webs and the functional traits of keystone species. Integrative and Comparative Biology, 57(5), 961-976.

https://doi.org/10.1093/icb/icx119

Koo, T. K., \& Li, M. Y. (2016). A guideline of selecting and reporting intraclass correlation coefficients for reliability research. Journal of Chiropractic Medicine, 15(2), 155-163. https://doi.org/10.1016/j.jcm.2016.02.012

Lang, T., \& McKee, M. (2018). Brexit poses serious threats to the availability and affordability of food in the United Kingdom. Journal of Public Health, 40(4), e608-e610. https://doi.org/10.1093/pubmed/fdy073

Li, N. P., van Vugt, M., \& Colarelli, S. M. (2018). The evolutionary mismatch hypothesis: Implications for psychological science. Current Directions in Psychological Science, 27(1), 38-44. https://doi.org/10.1177/0963721417731378

Lima, S. L. (1986). Predation risk and unpredictable feeding conditions: Determinants of body mass in birds. Ecology, 67(2), 377-385. https://doi.org/10.2307/1938580

Long, L. (2020). Hemingway editor. http://www.hemingwayapp.com/

Lufkin, B. (2020). Coronavirus: The psychology of panic buying. https://www.bbc.com/worklife/article/20200304-coronavirus-covid-19-update-whypeople-are-stockpiling

Marteau, T. M., \& Bekker, H. (1992). The development of a six-item short-form of the state scale of the Spielberger State - Trait Anxiety Inventory (STAI). British Journal of Clinical Psychology, 31(3), 301-306. https://doi.org/10.1111/j.2044-8260.1992.tb00997.x

Merriam-Webster. (2021). Insecurity. Merriam-Webster.com dictionary. Retrieved February 23, 2021, from https://www.merriam-webster.com/dictionary/insecure 
Mokken, R. J. (1971). A theory and procedure of scale analysis: With applications in political research. De Gruyter Mouton. https://doi.org/10.1515/9783110813203

Nettle, D. (2017). Does hunger contribute to socioeconomic gradients in behavior? Frontiers in Psychology, 8, 358. https://doi.org/10.3389/fpsyg.2017.00358

Nettle, D., Andrews, C., \& Bateson, M. (2017). Food insecurity as a driver of obesity in humans: The insurance hypothesis. Behavioral and Brain Sciences, 40. https://doi.org/10.1017/S0140525X16000947

Nettle, D., Johnson, E., Johnson, M., \& Saxe, R. (2020). Why has the COVID-19 pandemic increased support for Universal Basic Income? https://doi.org/10.31234/osf.io/csr3u

Nettle, D., Joly, M., Broadbent, E., Smith, C., Tittle, E., \& Bateson, M. (2019). Opportunistic food consumption in relation to childhood and adult food insecurity: An exploratory correlational study. Appetite, 132, 222-229. https://doi.org/10.1016/j.appet.2018.07.018

Ornstein, R. E., \& Ehrlich, P. R. (2000). New world new mind: Moving toward conscious evolution. ISHK.

Otterbring, T., \& Sela, Y. (2020). Sexually arousing ads induce sex-specific financial decisions in hungry individuals. Personality and Individual Differences, 152, 109576. https://doi.org/10.1016/j.paid.2019.109576

Petersen, M. B., Aarøe, L., Jensen, N. H., \& Curry, O. (2014). Social welfare and the psychology of food sharing: Short-term hunger increases support for social welfare. Political Psychology, 35(6), 757-773. https://doi.org/10.1111/pops.12062

Podsakoff, P. M., MacKenzie, S. B., Lee, J.-Y., \& Podsakoff, N. P. (2003). Common method biases in behavioral research: A critical review of the literature and recommended remedies. Journal of Applied Psychology, 88(5), 879-903. https://doi.org/10.1037/0021-9010.88.5.879 
Radimer, K. L., Olson, C. M., \& Campbell, C. C. (1990). Development of indicators to assess hunger. The Journal of Nutrition, 120 (Suppl 11), 1544-1548. https://doi.org/10.1093/jn/120.suppl_11.1544

Rosseel, Y. et al. (2012). Lavaan: An R package for structural equation modeling. Journal of Statistical Software, 48(i02). https://doi.org/10.18637/jss.v048.i02

Rotter, J. B. (1966). Generalized expectancies for internal versus external control of reinforcement. Psychological Monographs: General and Applied, 80(1), 1-28. https://doi.org/10.1037/h0092976

Saint Ville, A., Po, J. Y. T., Sen, A., Bui, A., \& Melgar-Quiñonez, H. (2019). Food security and the Food Insecurity Experience Scale (FIES): Ensuring progress by 2030. Food Security, 11, 483-491. https://doi.org/10.1007/s12571-019-00936-9

Sherman, M. A. (1973). Bound to be easier? The negative prefix and sentence comprehension. Journal of Verbal Learning and Verbal Behavior, 12(1), 76-84. https://doi.org/10.1016/S0022-5371(73)80062-3

Shrout, P. E., \& Fleiss, J. L. (1979). Intraclass correlations: Uses in assessing rater reliability. Psychological Bulletin, 86(2), 420-428. https:/ /doi.org/10.1037/0033-2909.86.2.420

Sijtsma, K., \& Molenaar, I. W. (2002). Introduction to nonparametric item response theory (Vol. 5). Sage.

Song, H., \& Schwarz, N. (2008). If it's hard to read, it's hard to do: Processing fluency affects effort prediction and motivation. Psychological Science, 19(10), 986-988. https://doi.org/10.1111/j.1467-9280.2008.02189.x

Sova, C., Flowers, K., \& Man, C. (2019). Climate change and food security: A test of U.S. leadership in a fragile world. https://www.csis.org/analysis/climate-change-andfood-security-test-us-leadership-fragile-world 
Stoet, G. (2010). PsyToolkit: A software package for programming psychological experiments using Linux. Behavior Research Methods, 42(4), 1096-1104. https://doi.org/10.3758/BRM.42.4.1096

Stoet, G. (2017). PsyToolkit: A novel web-based method for running online questionnaires and reaction-time experiments. Teaching of Psychology, 44 (1), 24-31. https://doi.org/10.1177/0098628316677643

Thompson, E. R. (2007). Development and validation of an internationally reliable short-form of the Positive and Negative Affect Schedule (PANAS). Journal of Cross-Cultural Psychology, 38(2), 227-242. https://doi.org/10.1177/0022022106297301

Tingley, D., Yamamoto, T., Hirose, K., Keele, L., \& Imai, K. (2014). Mediation: R package for causal mediation analysis. Journal of Statistical Software, 59(5), 1-38. https://doi.org/10.18637/jss.v059.i05

Tooby, J., \& Cosmides, L. (1990). The past explains the present: Emotional adaptations and the structure of ancestral environments. Ethology and Sociobiology, 11(4-5), 375-424. https://doi.org/10.1016/0162-3095(90)90017-Z

Twenge, J. M. (2000). The age of anxiety? the birth cohort change in anxiety and neuroticism, 1952-1993. Journal of Personality and Social Psychology, 79(6), 1007. https://doi.org/10.1037/0022-3514.79.6.1007

Umanath, S., \& Coane, J. H. (2020). Face validity of remembering and knowing: Empirical consensus and disagreement between participants and researchers. Perspectives on Psychological Science, 15(6), 1400-1422. https://doi.org/10.1177/1745691620917672 UNDP. (2020). Goal 12: Responsible consumption and production. https://www.undp.org/content/undp/en/home/sustainable-developmentgoals/goal-12-responsible-consumption-and-production.html

Van der Ark, L. A. (2007). Mokken scale analysis in R. Journal of Statistical Software, 20(11), 1-19. https://doi.org/10.18637/jss.v020.i11 
Van der Ark, L. A. (2012). New developments in mokken scale analysis in R. Journal of Statistical Software, 48(5), 1-27. https://doi.org/10.18637/jss.v048.i05

Van Schuur, W. H. (2003). Mokken scale analysis: Between the Guttman scale and parametric item response theory. Political Analysis, 11(2), 139-163. https://doi.org/10.1093/pan/mpg002

Watson, D., Clark, L. A., \& Tellegen, A. (1988). Development and validation of brief measures of positive and negative affect: The PANAS Scales. Journal of Personality and Social Psychology, 54 (6), 1063-1070.

Webb, P., Coates, J., Frongillo, E. A., Rogers, B. L., Swindale, A., \& Bilinsky, P. (2006). Measuring household food insecurity: Why it's so important and yet so difficult to do. The Journal of Nutrition, 136(5), 1404S-1408S.

https://doi.org/10.1093/jn/136.5.1404S

Wheeler, T., \& Von Braun, J. (2013). Climate change impacts on global food security. Science, 341(6145), 508-513. https://doi.org/10.1126/science.1239402 